\title{
Variational Approach to Tomographic Reconstruction
}

\author{
Jan Kybic, Thierry Blu, and Michael Unser \\ Biomedical Imaging Group, DMT/IOA \\ Swiss Federal Institute of Technology Lausanne \\ CH-1015 Lausanne EPFL, Switzerland
}

\begin{abstract}
We formulate the tomographic reconstruction problem in a variational setting. The object to be reconstructed is considered as a continuous density function, unlike in the pixel-based approaches. The measurements are modeled as linear operators (Radon transform), integrating the density function along the ray path. The criterion that we minimize consists of a data term and a regularization term. The data term represents the inconsistency between applying the measurement model to the density function and the real measurements. The regularization term corresponds to the smoothness of the density function.

We show that this leads to a solution lying in a finite dimensional vector space which can be expressed as a linear combination of generating functions. The coefficients of this linear combination are determined from a linear equation set, solvable either directly, or by using an iterative approach.

Our experiments show that our new variational method gives results comparable to the classical filtered backprojection for high number of measurements (projection angles and sensor resolution). The new method performs better for medium number of measurements. Furthermore, the variational approach gives usable results even with very few measurements when the filtered back-projection fails. Our method reproduces amplitudes more faithfully and can cope with high noise levels; it can be adapted to various characteristics of the acquisition device.
\end{abstract}

Keywords: tomography, reconstruction, variational, filtered back-projection

\section{INTRODUCTION}

Tomographic reconstruction is the process of reconstructing an object or its cross section from several images of its (transaxial) projections. ${ }^{1}$ Its applications are numerous. The simplest one is the reconstruction in transmission tomography, when the object is illuminated by a parallel beam of rays. The signal is attenuated by the object. If we neglect the scatter, then the logarithm of the intensity of the received signal is proportional to the integral of the absorption along the beam path. Typical examples are the kinds of tomography performed in X-ray CT scanners. This image modality is called transmission tomography. It is used in medical imaging and for non-destructive testing of mechanical objects. Another application of tomographic reconstruction is reflection tomography. An example is radar imaging, where the received signal amplitude is proportional to the integral of the reflectivity of the object in the area sensed by the camera. Yet another example from the medical domain is emission tomography, for example positron emission tomography (PET). Here, radioactive isotopes are introduced in the body and the radiation emitted by their decay is detected. The rays from different directions are separated by colimators. Under the assumption that the scatter and absorption in the tissue can be neglected, the signal received is proportional to the density of the radioactive particles along the beam path.

\subsection{Radon transform}

Mathematically, the integral transformation from an object to the set of projections is called the Radon transform. The Radon transform of a $2 \mathrm{D}$ function $f(x, y)$ is another two-parameter function $F(\theta, u)=(\mathscr{R} f)(\theta, u)$, where the parameter $\theta$ represents an angle and the parameter $u$ a distance from the origin. The Radon transform is defined by the ray integral ${ }^{1}$ :

$$
F(\theta, u)=(\mathscr{R} f)(\theta, u)=\int f\left(t \cos \theta-u_{j} \sin \theta, t \sin \theta+u_{j} \cos \theta\right) \mathrm{d} t
$$

The function $F$ is often called a 'sinogram' because the image of a single point becomes a sinusoid.

Correspondence: email: Jan.Kybic@epfl.ch 


\subsection{Filtered back-projection}

The most often used reconstruction method is filtered back-projection. ${ }^{1,2}$ The basic idea is to back-project all the ray-integrals in the object space. In other words, for each point $(x, y)$ in the object space, one accumulates the contribution of all the ray integrals whose path intersects the point $(x, y)$. Furthermore, before back-projecting, each projection (the sinogram $F$ for a fixed angle $\theta$ ) is prefiltered by a unidimensional ramp filter with frequency response $|\omega|$, which accounts for the fact that the back-projection is only an adjoint, not the inverse of the Radon transform.

\subsection{Problems of filtered back-projection}

The filtered back-projection algorithm is the exact inverse of the forward Radon transform, provided that the sinogram $F$ is known continuously. That is, we need to have an infinite number of projection angles and an infinite number of measures for each angle. Consequently, the reconstruction quality drops dramatically when only a limited amount of samples is available. Second, the derivative nature of the ramp filter makes it prone to noise. Therefore, in practical implementations, the ideal ramp filter is usually replaced by its windowed approximation, such as Ram-Lak, or Shepp-Logan filters. ${ }^{1}$ The window size, however, is a compromise between the noise sensitivity and resolution. Third, filtered back-projection is directly applicable only for uniform and regular sampling geometry. When the rays are not parallel (which is typical for modern fan-beam scanners), or the data from some projection angles is missing, some pre- or post-processing needs to by applied.

\subsection{Algebraic methods}

It is also possible to formulate the tomographic reconstruction as a general image reconstruction problem. ${ }^{1,2}$ The resulting family of reconstruction method is traditionally called ART (algebraic reconstruction techniques). We represent the object as a linear combination of basis functions, typically pixels, with some unknown coefficients $a_{i}$. Other basis functions, such as 'natural pixels'3 are also used. Then the observations (the samples of the sinogram) can be expressed as a linear combination of the same coefficients $a_{i}$. To find the coefficients $a_{i}$, which amounts to reconstructing the object, one solves a linear system of equations. Two scenarios are possible. If enough measures are available, one has an over-determined system, which is solved in the least-squares sense. This also helps to account for the noise. If, on the other hand, there is not enough measures in some region to determine the coefficient values, one is faced with an under-determined problem. In this case, one solves the regularized version of the problem which supplies the additional constraints.

Given the large number of unknowns (one for every basis function or pixel), direct solution is usually not feasible. However, as each observation is influenced only by the pixels on the corresponding beam path, the equivalent system matrix is sparse. Therefore, iterative linear solvers can be applied. ${ }^{2,4,5}$

The advantage of the algebraic method is that it is directly applicable to an arbitrary scanning geometry. On the other hand, only objects representable using the basis functions (pixels) can be reconstructed. Therefore, to represent objects well, many basis functions must be used. The resulting system of linear equations is huge and thus slow to solve.

\section{VARIATIONAL APPROACH}

In this article, we propose an alternative, variational approach to tomographic reconstruction, to remedy the various disadvantages of the filtered back-projection. In terms of philosophy and of the resulting algorithm, this approach is close to the algebraic methods with one important difference; namely, the type of basis functions which are not specified beforehand, but which are derived mathematically as the solution of the optimization problem. To explain the motivation of the variational approach, let us first consider a simple example.

\subsection{Motivation for variational reconstruction}

As illustrative example, we consider the task of interpolating a unidimensional function given its values at some sampling points. As illustrated in Figure 1, there is an infinity of functions passing through the given points. Nevertheless, most people would probably agree that the smooth approximation curve in Figure 1 looks 'more correct' than the rugged noisy approximation. Similarly, we can often quantify the degree of plausibility of a function for a given application. Then, we search for the most plausible function satisfying our interpolation (consistency) conditions. This is the key concept behind our approach. From now on, we will concentrate on the typical case 


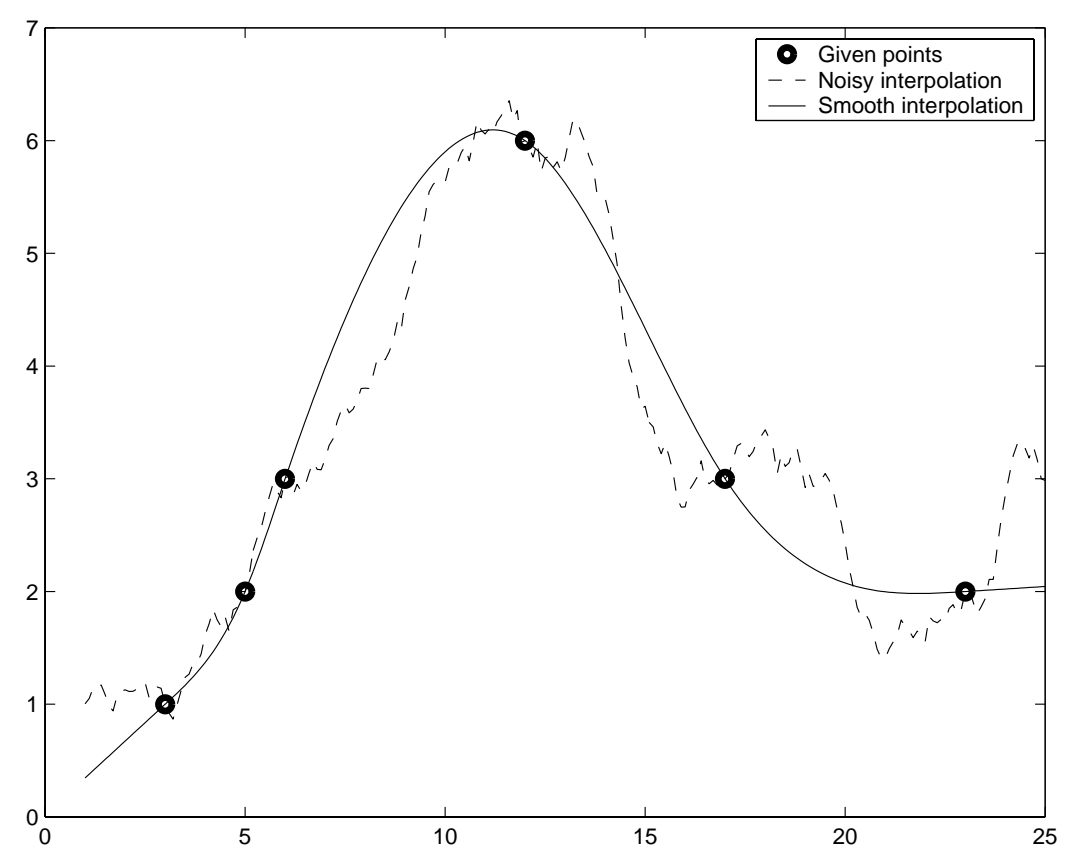

Figure 1. When interpolating a function from its values (circles), many solutions are possible. However, smooth interpolation (solid line) is usually preferable to a more rugged one (dashed line).

where we want the solution to be 'smooth'. As smoothness can be measured by the amplitude of the derivatives, maximizing smoothness translates into minimizing the norm of a differential operator.

In Figure 1 the smooth curve minimizes the $L_{2}$-norm of the second derivative $\left\|f^{\prime \prime}\right\|_{L_{2}}$, which is known to yield a cubic spline interpolation. ${ }^{6,7}$ This mathematical result also leads to the functional form of the solution which can be expressed as a linear combination of basis functions; e.g., cubic B-splines. The solution of the interpolation problem is then computed by fitting a linear combination of basis functions to the data.

\subsection{Tomographic reconstruction as a variational problem}

Similarly to our example above, we consider the unknown cross-section of the object to be reconstructed as a scalar function of $f(x, y)$, where the two continuous parameters $x$ and $y$ represent spatial coordinates. We project the object using projection angles $\theta_{1}, \theta_{2}, \ldots, \theta_{N}$. Furthermore, we sample each projection at distances $u_{1}, u_{2}, \ldots, u_{q}$. Thus, we have $q \times N$ scalar measures described by the Radon transform equation.

$$
s_{i j}=\mathscr{R} f\left(\theta_{i}, u_{j}\right)=\int f\left(t \cos \theta_{i}-u_{j} \sin \theta_{i}, t \sin \theta_{i}+u_{j} \cos \theta_{i}\right) \mathrm{d} t
$$

Similarly to the unidimensional example case, there is an infinity of functions $f$ for which equation (2) holds. Therefore, we introduce a regularization criterion $J(f)$ which will characterize the plausibility of a specific function $f$. In the context of biomedical imaging, it makes sense to assume that the function $f$ is smooth. In this case, a suitable plausibility criterion is the Duchon's norm ${ }^{8}$ measuring the amplitude of the second partial derivatives as follows:

$$
J(f)=\int\left(\frac{\partial^{2} f}{\partial x^{2}}\right)^{2}+2\left(\frac{\partial^{2} f}{\partial x \partial y}\right)^{2}+\left(\frac{\partial^{2} f}{\partial y^{2}}\right)^{2} \mathrm{~d} x \mathrm{~d} y
$$

The implications of choosing this norm are explained in Section 3.5. Here we only point to the fact that if $f$ is a linear polynomial of the type $f(x, y)=a_{1} x+a_{2} y+a_{3}$, then the corresponding criterion is zero, $J(f)=0$. 


\subsection{Bilinear form}

The criterion (3) can be expressed as

$$
J(f)=B(f, f)
$$

where $B$ is a bilinear form (a scalar functional form linear in both its arguments) given by the following formula:

$$
B(f, g)=\int \frac{\partial^{2} f}{\partial x^{2}} \frac{\partial^{2} g}{\partial x^{2}}+2 \frac{\partial^{2} f}{\partial x \partial y} \frac{\partial^{2} g}{\partial x \partial y}+\frac{\partial^{2} f}{\partial y^{2}} \frac{\partial^{2} g}{\partial y^{2}} \mathrm{~d} x \mathrm{~d} y
$$

It satisfies the Cauchy-Schwartz inequality

$$
|B(f, g)| \leq J(f) J(g)
$$

\subsection{Region of interest}

The reconstruction problem as we have described it so far considers the object function at the entire plane $\mathbb{R}^{2}$. It also considers the sampling operators (2) on the entire plane. However, this leads to an ill-posed problem because we try to reconstruct an infinite plane using a finite number of coarsely placed measuring rays. In the absence of information about the evolution of $f$ far from the origin, the reconstruction optimal in the sense of (3) is linear in each direction and its measure using (2) is therefore either zero or infinity. As a consequence, there is no reconstruction consistent with the measures minimizing (3).

Furthermore, considering the entire plane does not correspond to the physical reality, because normally the detectors are directional and only rays coming from the interior of the tomographic apparatus are taken into account. Therefore, we choose to apply the sampling operators as well as the regularization criterion in a region of interest $S$ of a limited spatial extend. We specifically chose a circle of a radius $R$ :

$$
S=\left\{(x, y) \in \mathbb{R}^{2} ; x^{2}+y^{2} \leq R^{2}\right\}
$$

This modifies the equations (2), (3), and (5) as follows:

$$
\begin{aligned}
s_{i j} & =\mathscr{R} f\left(\theta_{i}, u_{j}\right)=\int_{S} f(\underbrace{t \cos \theta_{i}-u_{j} \sin \theta_{i}}_{x}, \underbrace{t \sin \theta_{i}+u_{j} \cos \theta_{i}}_{y}) \mathrm{d} t \\
J(f) & =\int_{S}\left(\frac{\partial^{2} f}{\partial x^{2}}\right)^{2}+2\left(\frac{\partial^{2} f}{\partial x \partial y}\right)^{2}+\left(\frac{\partial^{2} f}{\partial y^{2}}\right)^{2} \mathrm{~d} x \mathrm{~d} y \\
B(f, g) & =\int_{S} \frac{\partial^{2} f}{\partial x^{2}} \frac{\partial^{2} g}{\partial x^{2}}+2 \frac{\partial^{2} f}{\partial x \partial y} \frac{\partial^{2} g}{\partial x \partial y}+\frac{\partial^{2} f}{\partial y^{2}} \frac{\partial^{2} g}{\partial y^{2}} \mathrm{~d} x \mathrm{~d} y
\end{aligned}
$$

Consequently, we will consider $f$ only inside $S$.

\section{SOLUTION OF THE VARIATIONAL RECONSTRUCTION PROBLEM}

The variational problem of minimizing $J$ given by (9) under constraints (8) can be solved by the Lagrange multipliers method introducing an extended criterion:

$$
J^{*}(f, \boldsymbol{\lambda})=J(f)+2 \sum \lambda_{i j}\left(s_{i j}-\mathscr{R} f\left(\theta_{i}, u_{j}\right)\right)
$$

where $\boldsymbol{\lambda}$ is the vector of all $\lambda_{i j}$. The Lagrange theorem tells us that $f$ solves the original constrained minimization problem if there is some $\boldsymbol{\lambda}$, such that $(f, \boldsymbol{\lambda})$ is a saddle point of $J^{*}$. We carry on using the standard variational argument. We take a small perturbation $g$. We calculate the first order variation of $J^{*}$ with respect to $g$. This variation has to be zero, if $(f, \boldsymbol{\lambda})$ is to be a saddle point.

$$
0=J^{*}(f+g)-J^{*}(f)=2 B(f, g)-2 \sum_{i j} \lambda_{i j} \mathscr{R} g\left(\theta_{i}, u_{j}\right)
$$


We have used the fact that $J(f)=B(f, f)$ and the linearity of $B$. As the variation (12) needs to be zero, this implies that

$$
B(f, g)=\sum_{i j} \lambda_{i j} \mathscr{R} g\left(\theta_{i}, u_{j}\right)
$$

for an arbitrary function $g$ such that $J(g)<\infty$ (otherwise the criterion $J(f+g)$ does not make sense). A consequence of the Cauchy-Schwartz inequality (6) is that for any linear polynomial $p=a_{1} x+a_{2} y+a_{3}$, we have $B(f, p)=0$ and thus from (13), we get so-called orthogonality conditions

$$
0=\sum_{i j} \lambda_{i j} \mathscr{R} p\left(\theta_{i}, u_{j}\right)
$$

Suppose now that we have a set of functions $\varphi_{i j}$ which satisfy the following equation

$$
B\left(\varphi_{i j}, g\right)=\mathscr{R} g\left(\theta_{i}, u_{j}\right) \text { for all } g, J(g)<\infty
$$

and which we will call fundamental solutions. Let us then take a function of the form

$$
f=\sum_{i j} \lambda_{i j} \varphi_{i j}+\underbrace{a_{1} x+a_{2} y+a_{3}}_{p}
$$

where $p$ is a linear polynomial so that $J(p)=0$. Such a function will always satisfy the condition (13). The function $f$ from (16) will therefore solve the reconstruction problem, provided that it satisfies the constraints (8) and that it is measurable in the sense of $(9)$, i.e., $J(f)<\infty$.

\subsection{Green function}

The remaining problem is now to find the fundamental solutions, also called Green functions. Substituting the explicit formulas (10) and (8) into (15) yields

$$
\int_{S} \frac{\partial^{2} \varphi_{i j}}{\partial x^{2}} \frac{\partial^{2} g}{\partial x^{2}}+2 \frac{\partial^{2} \varphi_{i j}}{\partial x \partial y} \frac{\partial^{2} g}{\partial x \partial y}+\frac{\partial^{2} \varphi_{i j}}{\partial y^{2}} \frac{\partial^{2} g}{\partial y^{2}} \mathrm{~d} x \mathrm{~d} y=\int_{S} g(\underbrace{t \cos \theta_{i}-u_{j} \sin \theta_{i}}_{x}, \underbrace{t \sin \theta_{i}+u_{j} \cos \theta_{i}}_{y}) \mathrm{d} t
$$

As finding the Green functions needs some rather complex mathematical notions, we will only present here the functions and show that they indeed satisfy the equation (17). For more information, we refer the reader to our forthcomming journal papers. ${ }^{9,10}$ A possible Green function for our reconstruction problem is:

$$
\varphi_{i j}(x, y)=\left|-x \sin \theta_{i}+y \cos \theta_{i}-u_{j}\right|^{3} / 12
$$

where 1/12 is a normalization constant. The shape of $\varphi$ is shown in Figure 2. Note that the manner in which the univariate function $|\eta|^{3}$ (where $\eta$ is a coordinate across the beam path) is extended to a bivariate $\varphi(x, y)$ by projecting it along the beam path is very similar to the 'natural pixel' concept. ${ }^{3}$

To prove that (18) satisfies (17), we first rotate the coordinate system, so that the privileged direction (the direction of integration along $t$ in (17)) coincides with one of the coordinate axis using the following substitution:

$$
\begin{aligned}
& \xi=x \cos \theta_{i}+y \sin \theta_{i}=t \\
& \eta=-x \sin \theta_{i}+y \cos \theta_{i}-u_{j}=0
\end{aligned}
$$

with inverse

$$
\begin{aligned}
& x=\xi \cos \theta_{i}-\left(\eta+u_{j}\right) \sin \theta_{i} \\
& y=\xi \sin \theta_{i}+\left(\eta+u_{j}\right) \cos \theta_{i}
\end{aligned}
$$



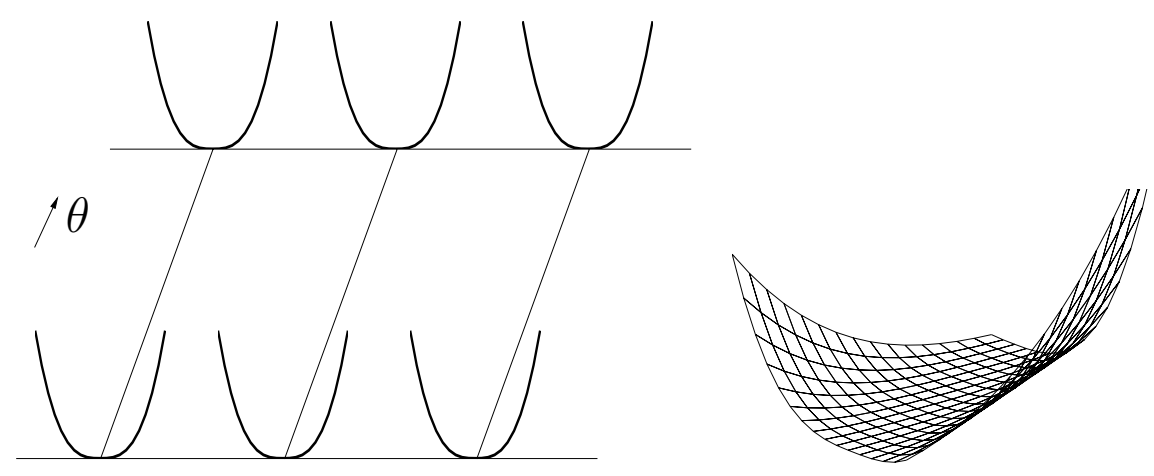

Figure 2. The Green function $\varphi$ as given by (18). On the left we see the univariate functions $|\eta|^{3}$ for three different shifts $u$ and how they are projected along the beam direction $\theta$. On the right we see the $3 \mathrm{D}$ view of one of those basis functions. Note its non-locality.

As the substitution is just a rotation and shift, its Jacobian is unitary which simplifies the substitution into (17). Moreover, as the integrand (differential operator) in the bilinear form $B$ is invariant to rotation and shift, the form of the left side of (17) does not change. The expression (17) in the new coordinates is

$$
\int_{S} \frac{\partial^{2} \varphi_{i j}}{\partial \xi^{2}} \frac{\partial^{2} g}{\partial \xi^{2}}+2 \frac{\partial^{2} \varphi_{i j}}{\partial \xi \partial \eta} \frac{\partial^{2} g}{\partial \xi \partial \eta}+\frac{\partial^{2} \varphi_{i j}}{\partial \eta^{2}} \frac{\partial^{2} g}{\partial \eta^{2}} \mathrm{~d} \xi \mathrm{d} \eta=\int_{S} g(\xi, \eta) \delta(\eta) \mathrm{d} \xi \mathrm{d} \eta
$$

where $g$ is now expressed in the new coordinates such that $g_{\xi \eta}(\xi, \eta)=g_{x y}(x, y)$ and Dirac's $\delta$ is used to go from $1 \mathrm{D}$ to $2 \mathrm{D}$ integral. The Green function $\varphi_{i j}$ from (18) in the new coordinates is

$$
\varphi_{i j}=|\eta|^{3} / 12
$$

The condition (7) for the region $S$ becomes

$$
S=\left\{(\xi, \eta) \in \mathbb{R}^{2} ; \xi^{2}+\left(\eta+u_{j}\right)^{2} \leq R^{2}\right\}
$$

Let us now substitute (24) into (23). As the partial derivatives of $\varphi_{i j}$ with respect to $\xi$ are obviously zero, the relation (23) simplifies to

$$
\int_{S} \frac{1}{2}|\eta| \frac{\partial^{2} g}{\partial \eta^{2}} \mathrm{~d} \xi \mathrm{d} \eta=\int_{S} g(\xi, \eta) \delta(\eta) \mathrm{d} \xi \mathrm{d} \eta
$$

We integrate $g$ over $\xi$ to get a new function $g_{\eta}$ :

$$
g_{\eta}(\eta)=\int_{\xi^{2} \leq R^{2}-\left(\eta+u_{j}\right)^{2}} g(\xi, \eta) \mathrm{d} \xi
$$

which yields a simplified form of (26)

$$
\frac{1}{2} \int_{-R-u_{j}}^{R-u_{j}}|\eta| \frac{\partial^{2} g_{\eta}}{\partial \eta^{2}} \mathrm{~d} \eta=g_{\eta}(0)
$$

Note that $g_{\eta}$ vanishes identically for the boundary points of the interval $\eta= \pm R-u_{j}$ because the integration in (27) is then carried out over an empty interval. This permits us to easily integrate (28) per-partes. The first application gives

$$
\frac{1}{2} \int_{-R-u_{j}}^{R-u_{j}}|\eta| \frac{\partial^{2} g_{\eta}}{\partial \eta^{2}} \mathrm{~d} \eta=-\frac{1}{2} \int_{-R-u_{j}}^{R-u_{j}}(\operatorname{sgn} \eta) \frac{\partial g_{\eta}}{\partial \eta} \mathrm{d} \eta
$$


The second application finally yields

$$
\frac{1}{2} \int_{-R-u_{j}}^{0} \frac{\partial g_{\eta}}{\partial \eta} \mathrm{d} \eta-\frac{1}{2} \int_{0}^{R-u_{j}} \frac{\partial g_{\eta}}{\partial \eta} \mathrm{d} \eta=\frac{1}{2}\left(-g_{\eta}\left(-R-u_{j}\right)+g_{\eta}(0)+g_{\eta}(0)-g_{\eta}\left(R-u_{j}\right)\right)=g_{\eta}(0)
$$

where we have again used the fact that $g_{\eta}$ vanishes at boundary points. Thus we have proved that $\varphi_{i j}$ as defined by (18) satisfies (17) and is thus the fundamental solution for our reconstruction problem.

\subsection{Linear equation system}

As we have seen, the solution of the variational reconstruction problem is given by (16) with fundamental solutions $\varphi_{i j}$ from (18). The remaining task is to determine the unknown coefficients $\lambda_{i j}$ and $a_{1}, a_{2}, a_{3}$ in (16). For this, we use the fact that the solution $f$ needs to fulfill the constraints (8). It also needs to satisfy the orthogonality constraints (14), which gives us three additional conditions by substituting $p=x, p=y$, and $p=1$ in (14). Together, the parameters can be determined from the following system of linear equations:

$$
\underbrace{\left[\begin{array}{ll}
\mathrm{A} & \mathrm{Q} \\
\mathrm{Q}^{T} & 0
\end{array}\right]}_{\mathrm{B}}\left[\begin{array}{l}
\lambda \\
\boldsymbol{a}
\end{array}\right]=\left[\begin{array}{l}
\boldsymbol{s} \\
\mathbf{0}
\end{array}\right]
$$

where $\boldsymbol{\lambda}$ is the vector of all $\lambda_{i j}, \boldsymbol{s}$ is the vector of all $s_{i j}$, and $\boldsymbol{a}=\left[a_{1} a_{2} a_{3}\right]^{T}$. The sub-matrix [A Q] corresponds to the constraints (8). The elements of $\mathrm{A}$ are the values of the measurement operator applied to the fundamental solutions

$$
(\mathrm{A})_{i j, k l}=\mathscr{R} \varphi_{k l}\left(\theta_{i}, u_{j}\right)
$$

The elements of $Q$ represent the contribution of the linear polynomial $p$ in (16) to (8). One line of $Q$ is

$$
(Q)_{i j}=\left[\begin{array}{lll}
(\mathscr{R} x)\left(\theta_{i}, u_{j}\right) & (\mathscr{R} y)\left(\theta_{i}, u_{j}\right) & (\mathscr{R} 1)\left(\theta_{i}, u_{j}\right)
\end{array}\right]
$$

It is interesting to note that the matrix $Q^{T}$ representing the orthogonality conditions (14) is exactly the transpose of Q.

Note that the size of the system matrix B grows linearly with the number of measures. Therefore, using standard linear equation system resolution techniques takes time proportional to the cube of the number of measures, which can be prohibitive for some applications. Faster techniques are being investigated.

\subsection{Radon transform of the fundamental solutions}

To assemble the matrix A, we need to calculate the Radon transform of the functions $\varphi_{k l}$ in (32). Substituting (18) and (8) into (32) yields

$$
\begin{aligned}
(\mathrm{A})_{i j, k l} & =\frac{1}{12} \int_{t^{2} \leq R^{2}-u_{l}^{2}}\left|-\sin \theta_{k}\left(t \cos \theta_{i}-u_{j} \sin \theta_{i}\right)+\cos \theta_{k}\left(t \sin \theta_{i}+u_{j} \cos \theta_{i}\right)-u_{l}\right|^{3} \mathrm{~d} t \\
& =\frac{1}{12} \int_{t^{2} \leq R^{2}-u_{l}^{2}}|t \underbrace{\sin \left(\theta_{i}-\theta_{k}\right)}_{\alpha}+\underbrace{u_{j} \cos \left(\theta_{i}-\theta_{k}\right)-u_{l}}_{\beta}|^{3} \mathrm{~d} t
\end{aligned}
$$

The integral can be calculated analytically using the following expression:

$$
\frac{1}{12} \int_{-T}^{T}|\alpha t+\beta|^{3} \mathrm{~d} t= \begin{cases}T|\beta|^{3} / 6 & \text { if } \alpha=0 \\ T \beta\left(\alpha^{2} T^{2}+\beta^{2}\right) / 6 & \text { if }-\alpha T+\beta>0 \\ -T \beta\left(\alpha^{2} T^{2}+\beta^{2}\right) / 6 & \text { if } \alpha T+\beta<0 \\ \left.\left((\alpha T+\beta)^{4}+(-\alpha T+\beta)^{4}\right) /(48|\alpha|)\right) & \text { otherwise }\end{cases}
$$


It is even easier to calculate the elements of $\mathrm{Q}$ given by (33).

$$
\begin{aligned}
& (\mathscr{R} x)\left(\theta_{i}, u_{j}\right)=\int_{t^{2} \leq R^{2}-u_{j}^{2}} t \cos \theta_{i}-u_{l} \sin \theta_{i} \mathrm{~d} t \\
& (\mathscr{R} y)\left(\theta_{i}, u_{j}\right)=\int_{t^{2} \leq R^{2}-u_{j}^{2}} t \sin \theta_{i}+u_{l} \cos \theta_{i} \mathrm{~d} t \\
& (\mathscr{R} 1)\left(\theta_{i}, u_{j}\right)=\int_{t^{2} \leq R^{2}-u_{j}^{2}} 1 \mathrm{~d} t
\end{aligned}
$$

All three are special cases of

$$
\int_{-T}^{T} \alpha t+\beta \mathrm{d} t=2 T \beta
$$

\subsection{Noisy measurements}

For real reconstruction problems, the measurements are always noisy. It turns out that the reconstruction method as described above is relatively sensitive to noise. That is to say that a small error in the measurements can influence the reconstructed solutions significantly. Therefore, we have opted for replacing the minimization of the criterion (9) with hard constraints (8) by a combined criterion with a least-squares data term

$$
J_{N}(f)=J(f)+\gamma \sum_{i j}(\underbrace{\mathscr{R} f\left(\theta_{i}, u_{j}\right)-s_{i j}}_{\varepsilon_{i j}})^{2}
$$

which we will minimize without constraints. This is equivalent to minimizing (9) with the constraint that the total square error $\sum_{i j} \varepsilon_{i j}^{2}$ is less or equal to a certain limit value.

Interestingly, the solution to the approximation problem of minimizing (41) has also the form (16), same as the constrained problem. Moreover, it can be shown that the parameters $\boldsymbol{\lambda}$ and $\boldsymbol{a}$ of the least-squares problem are given by the following linear system of equations

$$
\underbrace{\left[\begin{array}{cc}
\mathrm{A}+\gamma^{-1} \mathrm{I} & \mathrm{Q} \\
\mathrm{Q}^{T} & \mathbf{0}
\end{array}\right]}_{\mathrm{B}}\left[\begin{array}{c}
\boldsymbol{\lambda} \\
\boldsymbol{a}
\end{array}\right]=\left[\begin{array}{l}
\boldsymbol{s} \\
\mathbf{0}
\end{array}\right]
$$

which is essentially a regularized version of (31). This also demonstrates the expected fact that when $\gamma$ grows, the approximation problem solution tends to the solution of the constrained problem.

\subsection{Properties of the regularization criterion}

The regularization (plausibility) criterion (3) is invariant to rotations and translations. It is also pseudo-invariant to scaling (size changes); this means that the value of $J(f(\alpha x, \alpha y))$ changes with the scale $\alpha$ but independently of $f$. Similarly, $J$ is also pseudo-invariant with respect to the linear scaling of the values of $f$.

Consequently, the reconstruction is not influenced by the above mentioned transformations. This reflects the fact that we consider a reconstruction of an object equally plausible, regardless of its position, size, orientation, and amplitude.

These properties are to a large extent conserved even after the introduction of the region of interest $S$ in (9), provided that the object does not leave the region considered. 


\section{EXPERIMENTS AND FUTURE WORK}

Figure 3 shows a comparison of the reconstruction using the variational algorithm and classical filtered backprojection $^{1}$ as implemented in Matlab (by MathWorks, Inc.). We can observe that for a small number of measures, the variational reconstruction algorithm gives better result than filtered back-projection. The reconstruction using the variational method gives less blurring and more geometrical precision. Note also that the new algorithm, thanks to the consistency constraints, recovers well the amplitudes of the image, unlike filtered back-projection, for which the scale of the values of the reconstructed image is wrong by a large factor.

Currently, we are using a direct method to solve the linear system of equations (42). This obviously becomes impractical as the number of measurements grows. The next logical step will be to develop fast iterative solvers. We will also need to consider preconditioners because the basis functions (Green functions) are not localized, which makes the system of equations ill-conditioned. However, one clear advantage of working with the fundamental solutions as basis functions is that the form of the regularized system of equations (42) is especially simple; it differs from the exact system (31) by the mere addition of a diagonal term.

For a large number of measures, the results of the variational reconstruction are comparable to that of the filtered back-projection. In the present implementation, our variational method tends to become numerically instable and may require some tuning. Because of its higher computational complexity, one is probably better off using the filtered back-projection in the case of many measurements. However, due to the conceptual similarity of our representation with the natural pixel ART methods, ${ }^{3}$ we believe that similar techniques can be used to accelerate it. ${ }^{4}$

\section{CONCLUSIONS}

We have presented a novel approach to tomographic reconstruction based on variational principles. Its main advantages are the consistency of the reconstruction with the input data, and the optimality of the reconstruction in the sense of a user-defined criterion. It performs well even for a small number of measures, and can be applied to a computer tomography problems with arbitrary and even irregular geometry. The problem is solved exactly as presented; no approximations are carried out.

Our method bears some similarity to algebraic reconstruction techniques, except that we use a matched number of special basis functions which guarantee optimality in the sense of our criterion.

\section{REFERENCES}

1. A. K. Jain, Fundamentals of Digital Image Processing. Prentice Hall, 1989.

2. F. Natterer, The mathematics of computerized tomography. John Wiley \& Sons, 1986.

3. M. H. Buonocore, B. W. R., and A. Macovski, "A natural pixel decomposition for two-dimensional image reconstruction," IEEE Transactions on Biomedical Engineering, vol. 28, pp. 69-78, Feb. 1981.

4. G. T. Herman and L. B. Meyer, "Algebraic reconstruction techniques can be made computationally efficient," IEEE Transactions on Medical Imaging, vol. 12, pp. 600-609, Sept. 1993.

5. N. H. Clinthorne, T.-S. Pan, P.-C. Chiao, W. L. Rogers, and J. A. Stamos, "Preconditioning methods for improved convergence rates in iterative reconstruction," IEEE Transactions on Medical Imaging, vol. 12, Mar. 1993.

6. J. H. Ahlberg, E. N. Nilson, and J. L. Walsh, The theory of splines and their applications. New York: Academic Press, 1967.

7. I. Schoenberg, "Spline functions and the problem of graduation," Proc. Nat. Acad. Sci., vol. 52, pp. 947-950, 1964.

8. J. Duchon, "Splines minimizing rotation-invariant semi-norms in Sobolev spaces," in Constructive Theory of Functions of Several Variables (W. Schempp and K. Zeller, eds.), (Berlin), pp. 85-100, Springer-Verlag, 1977.

9. J. Kybic, T. Blu, and M. Unser, "Generalized sampling: A variational approach. Part I — Applications," IEEE Transactions on Signal Processing, 2001. In preparation.

10. J. Kybic, T. Blu, and M. Unser, "Generalized sampling: A variational approach. Part II — Theory," IEEE Transactions on Signal Processing, 2001. In preparation. 

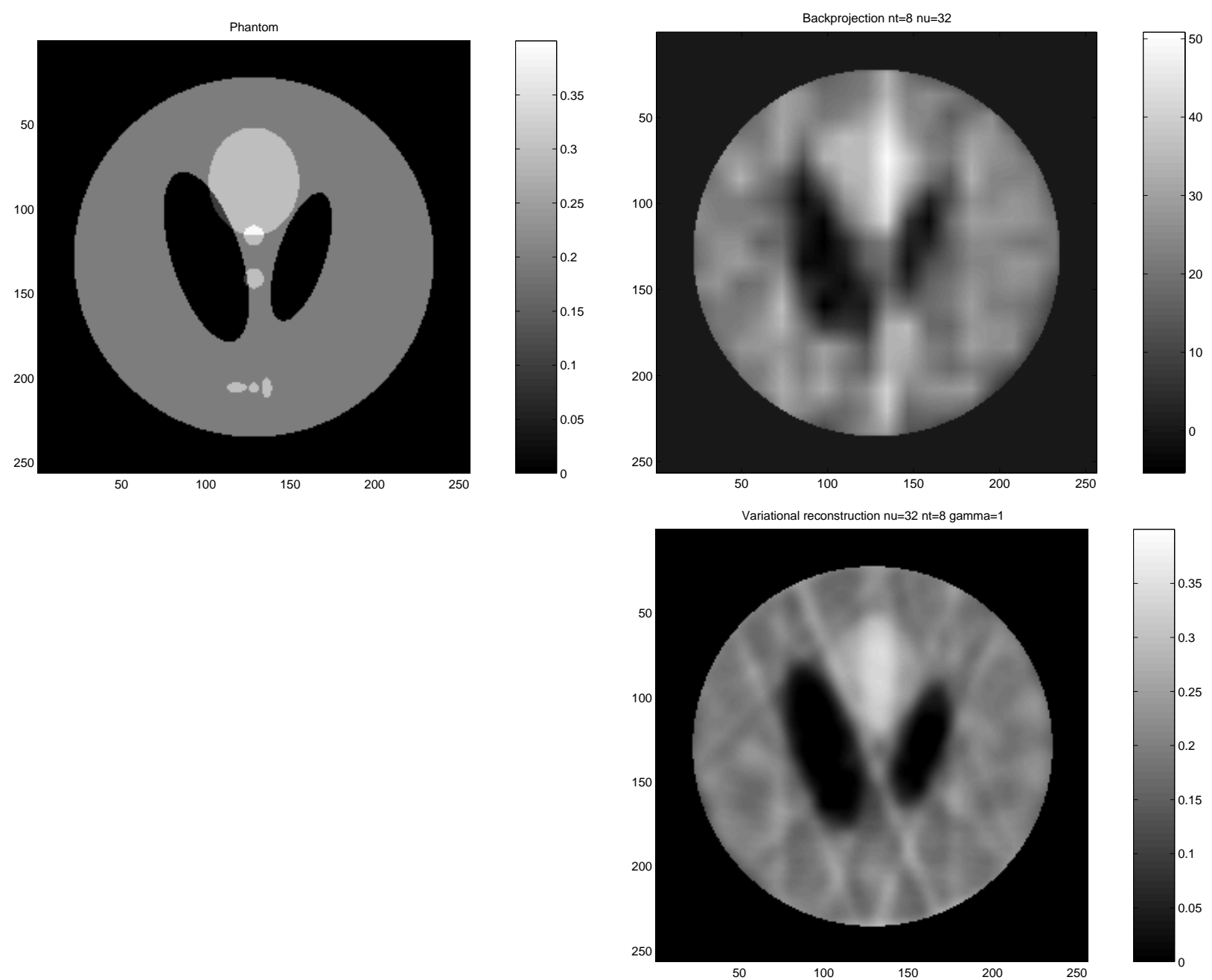

Figure 3. Reconstructiion of the inner part of the Shepp and Logan phantom ${ }^{1}$ (top left), using projections at 8 uniformly distributed angles, 32 equidistant measures per angle. The result of the filtered back-projection (top right) has more artifacts and is geometrically less precise than the variational reconstruction (bottom right). More importantly, the variational reconstruction recovers well the absolute amplitudes of the image, while the scale of the reconstruction using filtered back-projection is completely off. 